\title{
System Dynamics Simulation Model for Cardiovascular Heart Disease Risk Factors - Smoking and Alcohol Intake
}

\author{
Prof. (Dr.) Prem Pal Singh Tomar
}

Department of Electronics and Communication Engineering, Meerut Institute of Engineering and Technology, Meerut, India

\begin{abstract}
Detecting diseases at early stage can help to overcome and treat them accurately. Identifying the appropriate treatment depends on the method that is used in diagnosing the diseases. The incidence of cardiovascular heart disease (CVD) has been increasing steadily and so too its associated mortality. System Dynamics is appropriate methodology for Modelling and Simulation. The Expert knowledge about risk factors for CVD was elicited through interview and literature search. Two CVD risk factors Smoking and Alcohol Intake were analyzed by the proposed decision support system developed with System Dynamics Simulation software (iThink V9.0.2 ), used for the design, implementation and evaluation of the system. The proposed framework would be particularly useful for researchers in the field but also for medical practitioners and developers of medical decision support systems.
\end{abstract}

Keywords-Cardiovascular Heart Disease, System Dynamics Simulation, Smoking, Alcohol Intake, and Decision Support System.

\section{INTRODUCTION}

Cholesterol plays a major role in human heart health and high cholesterol is a leading risk factor for human cardiovascular disease. Cholesterol is a fatlike, waxy substance, which is used and found in tissues and plasma either as free cholesterol or in its storage form cholesterol ester. Cholesterol is essential for the survival [1]. Cholesterol is insoluble in water it is bound to blood lipoproteins for its transport. Cholesterol can be both good and bad. High-density lipoprotein (HDL) is good cholesterol and low-density lipoprotein (LDL) is bad cholesterol. High cholesterol in serum is a leading risk factor for human cardiovascular disease. The liver is responsible for the secretion of all other lipoproteins, most significantly VLDL. VLDL is secreted by the liver into the blood at an almost constant rate as a means of transport of synthesised fat and cholesterol to peripheral tissues. The lipoprotein lipase (LPL) enzyme hydrolyses triacylglycerol, degrades VLDL to IDL. A similar process leads from IDL to LDL. This is the lipoprotein cascade that transforms VLDL into IDL into LDL. HDL, is independently synthesized in the liver and is not a part of the lipoprotein cascade.) The density of LDL in the blood is determined by the rate of VLDL secreted from the liver. The unutilized excess cholesterol which is potentially harmful is scavenged from the tissues by HDL preventing its deposition and plaque formation [2].Hence, A higher level of HDL is an index of safety from the risk of Coronary Heart Disease (CHD).

Smoking: Cigarette smoking is an established coronary risk factor [3]. In the tables a smoker is defined as a person currently smoking at least one cigarette per day. Smoking affects the blood lipid profile: serum HDL and LDL cholesterol concentrations have been reported to be lower and higher, respectively, in smokers than in nonsmokers [4]. After cessation of smoking, HDL is observed to increase [5].

Alcohol Intake: Moderate alcohol intake is good as it raises the HDL by increasing esterified fraction of cholesterol in plasma [6]. The common perception of the effects of alcohol consumption on cardiovascular diseases has recently changed [7]. There is now evidence to suggest that light/moderate alcohol consumption protects against CHD and thrombotic stroke. However heavy consumption may be harmful as it increases blood pressure.

System dynamics is growing at an impressive exponential rate. System dynamics (SD) is a methodology involves development of causal diagrams and policy-oriented computer simulation models that are unique to each problem setting. The approach was developed by computer pioneer Jay W. Forrester in the mid-1950s and first described at length in his book Industrial Dynamics,[8] with some 
additional principles presented in later works [9][10][11][12]. The International System Dynamics Society was established in 1983, and within the society a special interest group on health issues was organized in 2003.

Simulation modeling has been applied in a number of health care situations [25] [26] [27] [28] and clearly has the potential to play a role in health care decision making at all levels. Simulation models provide quantitative information that provides a clear understanding of the problem thus enhancing decision making. Computer simulation provides a powerful tool that can be used to model and understand complex systems from the macro systems level to the micro genetic level [29].

Medical decision support system for risk assessment of CVD due to Smoking and/or Alcohol Intake, using System Dynamics Simulation software (iThink V9.0.2 ), is designed, implemented and evaluated.

\section{DESCRIPTION OF THE MODEL}

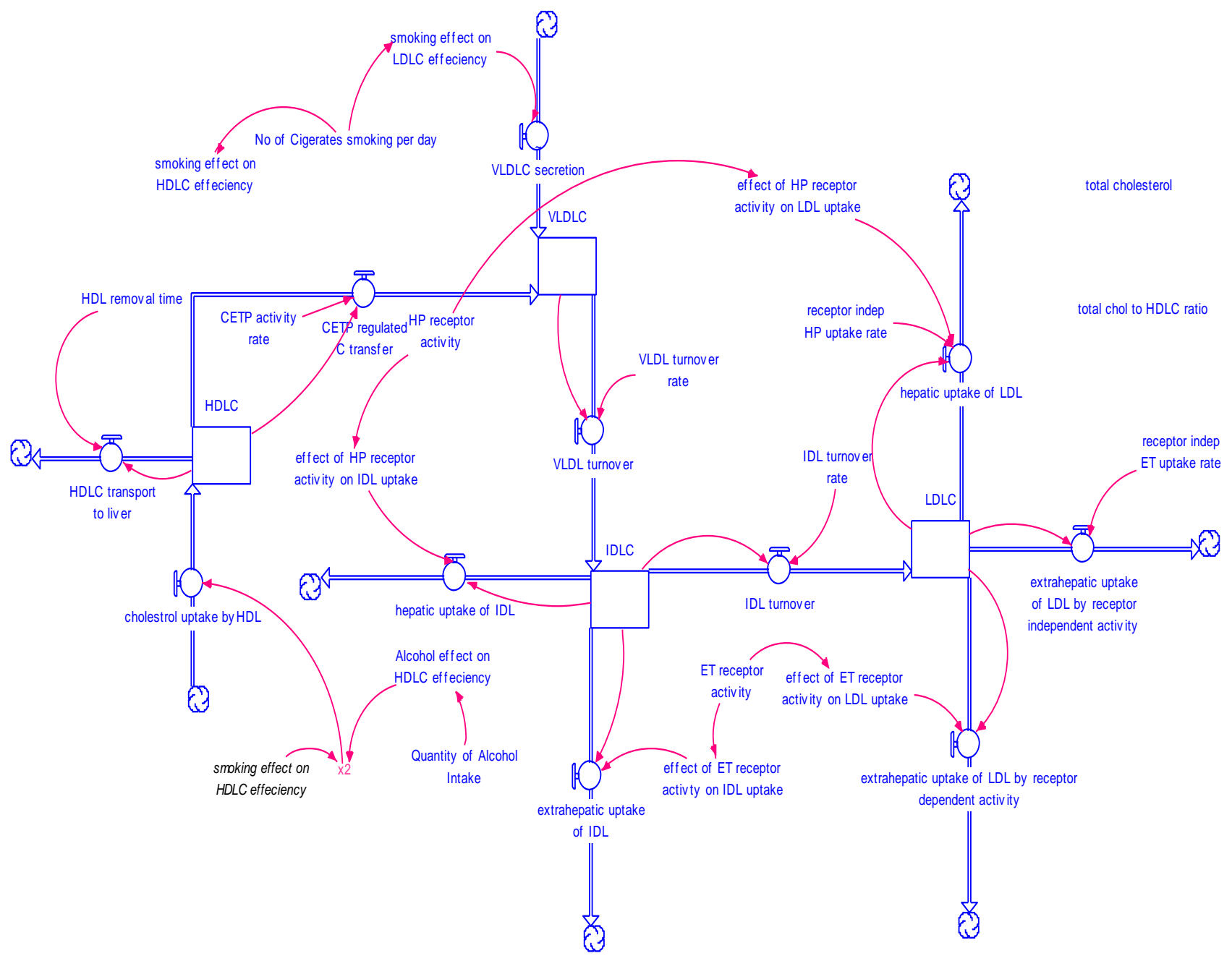

Fig.3.1: Causal Loop Diagram

VLDL is secreted from the liver for the purpose of transferring triacylglycerols to other tissues, mainly to the muscle and adipose tissue. After releasing most of its triacylglycerols, VLDL turns into intermediate density lipoprotein (IDL). About half of IDL are taken up by the liver and extrahepatic tissues, whereas the other half is converted to low density lipoprotein (LDL). Thus the level of cholesterol bound to LDL (LDLC) is mainly from the cascading process of VLDLC turning into IDLC and finally to LDLC, where VLDLC and IDLC stand for the level of 
cholesterol bound to VLDL and IDL respectively [13]. High density lipoproteins (HDL) are secreted from the steroidogenic tissues; mostly from the liver and the intestines. Main responsibility of $\mathrm{HDL}$ is to transfer cholesterol from extrahepatic cells to other lipoproteins, liver, and intestine. Movement of cholesteryl-ester to VLDL, IDL, and LDL is due to the activity of cholesterylester transfer protein or CETP. Half of the cholesterol bound to HDLs (HDLC) is taken up by steroidogenic tissues while the other half goes to the other lipoproteins via the stimulation by CETP [13].

Tobacco smoking is strongly and dose-dependently associated with all cardiovascular events, including CHD, stroke, peripheral arterial disease (PAD) and cardiovascular death [14]. Smoking cessation reduces these risks substantially, although the decrease is dependent on the duration of cessation [15]. Cigarette smoking potentiates the harmful effects of total cholesterol and reduces the cardioprotective properties of high density lipoprotein cholesterol (HDLC), increasing the risk of coronary heart disease (CHD) [3].

Alcohol consumption is associated with a reduced risk for coronary heart disease [16]. Alcohol intake results in an increase in HDL cholesterol levels. The functional consequences of this HDL increase are not fully understood at present [6].

There are four stocks in this sector. VLDLC, IDLC, and LDLC are connected in a cascaded form, while HDLC is related to these stocks via the flow CETP Regulated C Transfer. HDL cholesterol (HDLC) is increased with the HDL particles collecting cholesterol from extrahepatic tissues.

\section{FUNDAMENTAL ASSUMPTIONS FOR THE PROPOSED MODEL}

In the Casual Loop Diagram (CLD), figure 3.1,

1. The liver is assumed to be maintaining a constant level of HDL productivity. CETP related cholesterol transfer is also assumed to be occurring only between HDL and VLDL, excluded IDL and LDL as receivers of cholesterol into account.

2. Several studies [18] have shown that alcohol consumption does not affect CETP activities, and therefore no changes in CETP activity is made in the present study.

\section{SIMULATION RESULTS}

\section{Base Equilibrium Run}

In the model, screening of the patient/subject has borderline high blood cholesterol values, having weighs $74 \mathrm{~kg}$. www.ijaems.com
Keeping his diet (Caloric Intake) and exercise constant, he gets the same blood lipid levels throughout time and a total to HDL cholesterol ratio of 5.96. Since this ratio is quite high, he should consider some ways to lower his total cholesterol level and this ratio. His base level of VLDLC, IDLC, LDLC, and HDLC are $25 \mathrm{mg} / \mathrm{dL}, 18.57 \mathrm{mg} / \mathrm{dL}, 130$ $\mathrm{mg} / \mathrm{dL}$, and $35 \mathrm{mg} / \mathrm{dL}$. His total blood cholesterol is 208.6 $\mathrm{mg} / \mathrm{dL}$. The patient is initially assumed be non-smoker and non-drinker. The following (figure 4.1) simulation is obtained at equilibrium.

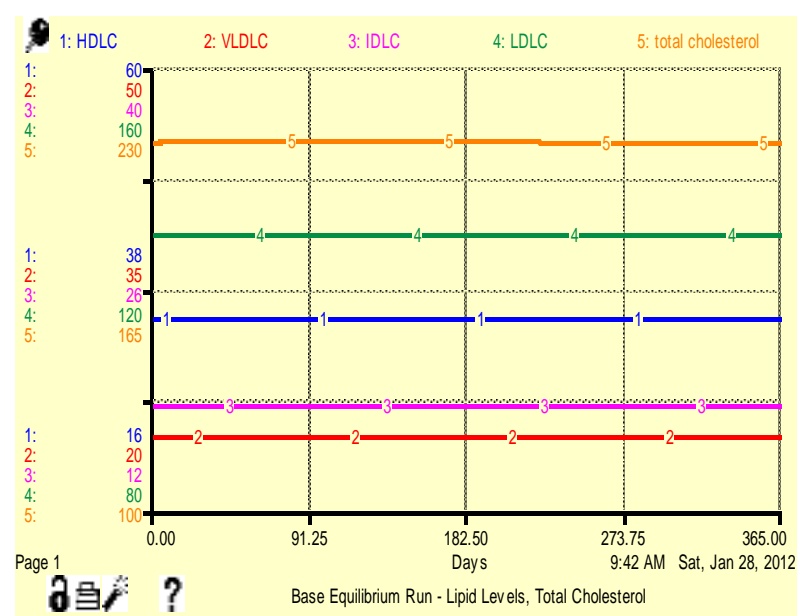

Fig.4.1: : Base Equilibrium Run-Lipid Cholesterol Levels, Total Cholesterol

\section{Smoking Effect Functions}

Cigarette smoking potentiates the harmful effects of total cholesterol and reduces the cardio-protective properties of high density lipoprotein cholesterol (HDLC), increasing the risk of coronary heart disease (CHD). Existing evidence suggests that every $1 \mathrm{mg} / \mathrm{dl}$ decrease in serum HDLC increases the risk of CHD by $2-3 \%$. Smoking affects the blood lipid profile: serum HDL and LDL cholesterol concentrations have been reported to be lower and higher, respectively, in smokers than in non-smokers [3] [17].

This function comprise of variable related to number of cigarette smoking per day. At the base level the person is assumed to be non-smoker.

In the experiment vs. Yin et al, for the subject smoking 10 cigarettes per day, results shows close values, HDLC Level as $30.22 \mathrm{mg} / \mathrm{dl}$ deceased from equilibrium level of $35 \mathrm{mg} / \mathrm{dl}$ and LDLC as $133.03 \mathrm{mg} / \mathrm{dl}$ increased from equilibrium level of $130 \mathrm{mg} / \mathrm{dl}$. These results can be seen in Figure 4.4. 


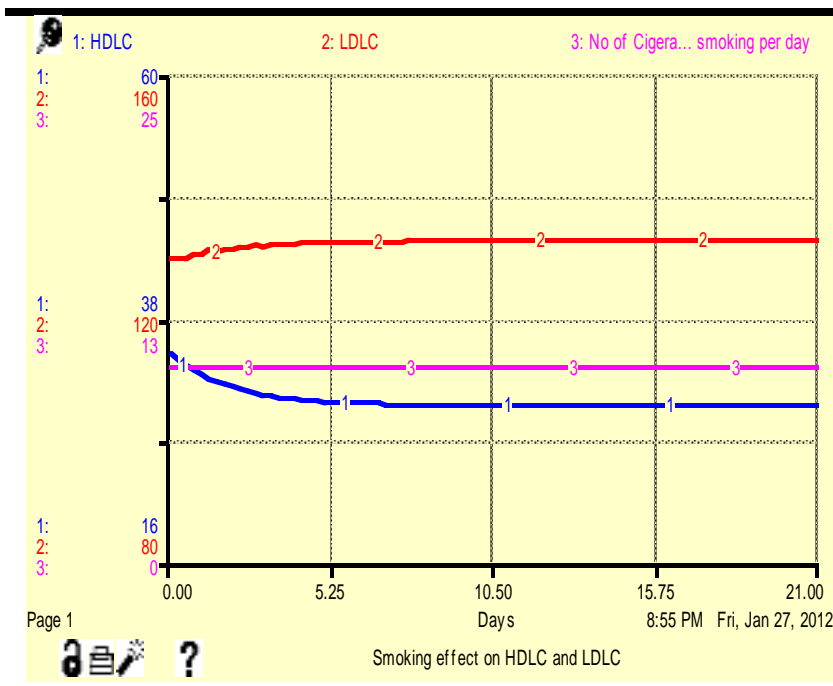

Fig.4.4: Smoking effect on HDLC and LDLC

\section{Alcohol Intake Effect Function}

Alcohol consumption is associated with increased HDL cholesterol levels. After experimenting on eleven healthy middle-aged men consumed four glasses ( $40 \mathrm{gm}$ of alcohol) per day for 3 weeks. It was observed that Alcohol consumption increased fasting HDL cholesterol by $13.6 \%$. The mechanism whereby alcohol increases HDL lipid levels is not clear. Both hepatic lipase and CETP activity may be involved [6][17].

These results of Alcohol effect on HDLC and LDLC can be seen in Figure 4.6.

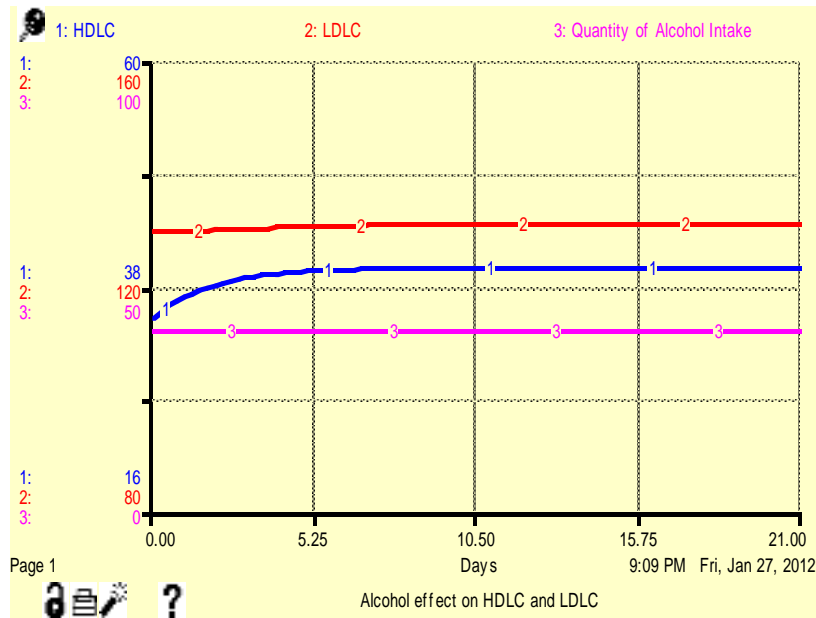

Fig.4.6: Alcohol effect on HDLC and LDLC

\section{SCENARIO ANALYSIS}

\section{Normal subject}

In the modal, the subject under consideration has borderline blood cholesterol levels; with base level of VLDLC, IDLC, LDLC, and HDLC are $25 \mathrm{mg} / \mathrm{dL}, 18.57 \mathrm{mg} / \mathrm{dL}, 130 \mathrm{mg} / \mathrm{dL}$, and $35 \mathrm{mg} / \mathrm{dL}$. His total blood cholesterol is $208.6 \mathrm{mg} / \mathrm{dL}$. The subject is initially assumed be non-smoker and non- drinker. He should try to find ways to lower them. In the following experiments

1. In the first scenario he tries to increase the desired Lipid Levels by smoking cessation

2. In the fifth scenario he consumes the moderate quantity of alcohol.

3. In the third scenario, observe under the effect of association of alcohol drinking and smoking on Lipid Levels.

\section{Smoking Cessation}

In this case the subject's exercises exercise and normal activity energy expenditure is $154 \mathrm{kcal}$ per day i.e. the base level. The literature reviews shows that smoking decreases and increases the HDLC Level and LDLC Level respectively. The subject is observed for 21 days, smoking 15 cigarette sticks per day. The results follow (figure 5.1and figure 5.2):

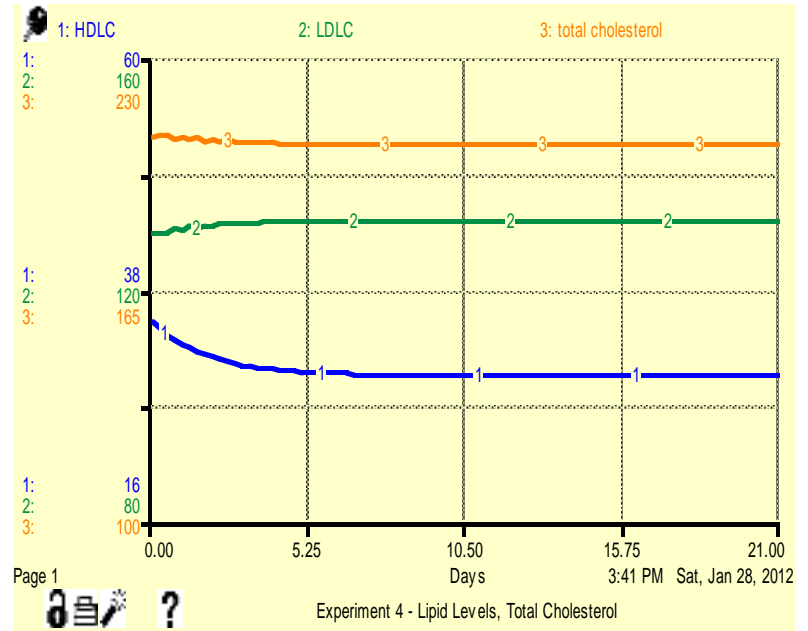

Fig.5.1: Smoking effect on Lipids Cholesterol Levels, Total Cholesterol.

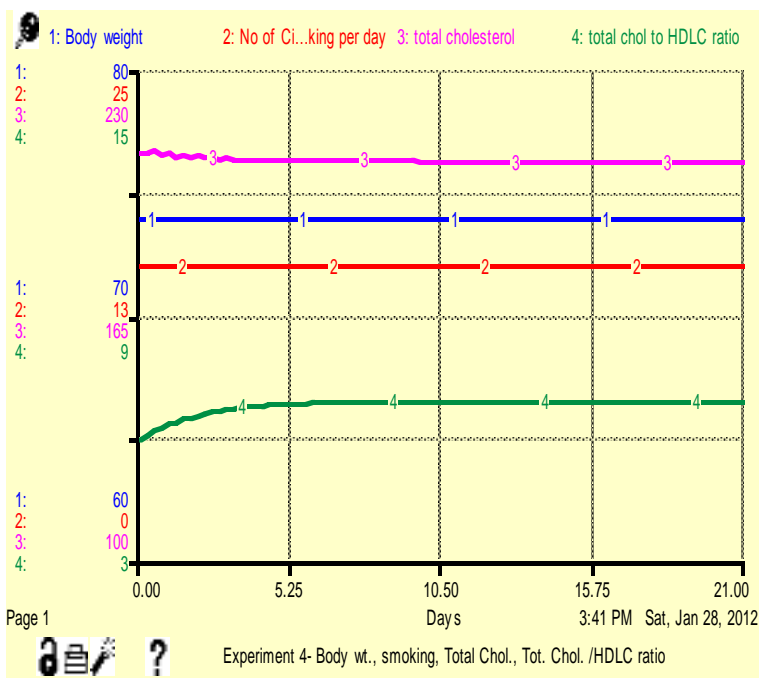

Fig.5.2: Smoking, Total Cholesterol and Cholesterol Ratio 
At the end of the simulation, his Total cholesterol to HDLC ratio increases to 6.9 from 5.9, and total cholesterol decreases by $2.36 \mathrm{mg} / \mathrm{dL}$ out of which $5.11 \mathrm{mg} / \mathrm{dL}$ is in HDLC i.e. total cholesterol is $206.24 \mathrm{mg} / \mathrm{dl}$ and HDLC is $29.89 \mathrm{mg} / \mathrm{dL}$. The simulation result indicated that the risk of CVD increases due to the rise in Total cholesterol to HDLC ratio and decrease in HDLC i.e. good cholesterol. Hence smoking cessation is a good option for such type of subject.

\section{Alcohol Intake}

In this case the subject's age is $\geq 21$ years and his exercises exercise and normal activity energy expenditure is $154 \mathrm{kcal}$ per day i.e. the base level. Several observational studies suggest that the moderate alcohol intake reduces the risk of cardiovascular disease. The major mechanism appears to the well known ability of alcohol to raise HDLC Level, however no significant changes has been noticed in LDLC Level. The subject is observed for 21 days, taking $40 \mathrm{~g}$ of wine per day. The results follow (figure 5.3 and figure 5.4):

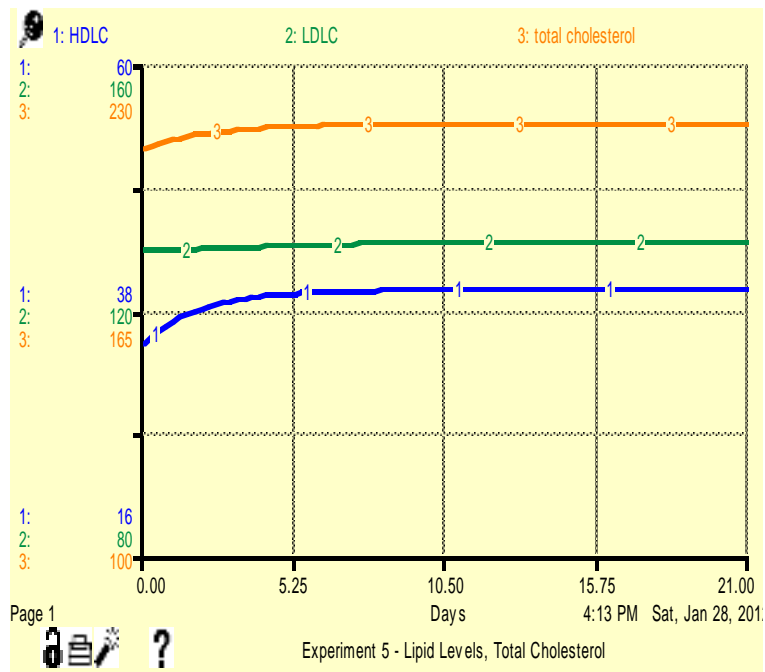

Fig.5.3: Alcohol intake effect on Lipids Cholesterol Levels, Total Cholesterol.

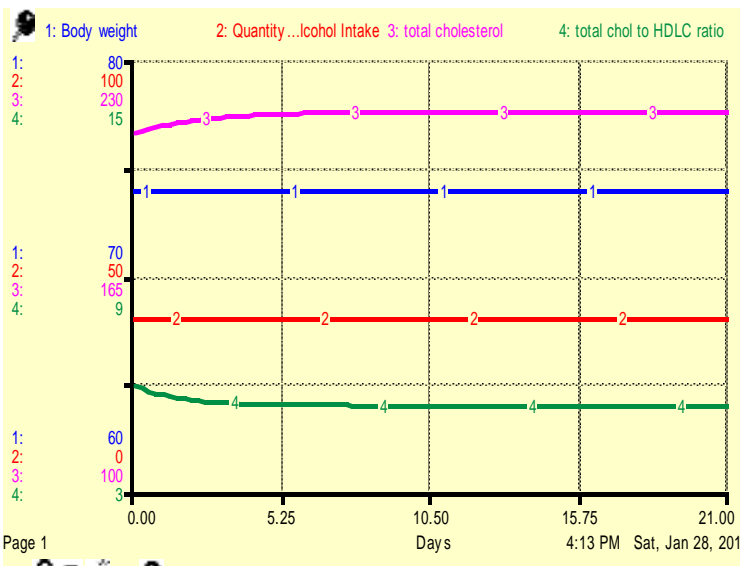

d当? Experiment 5- Body wt., Alcohol Intake, Total Chol., Tot. Chol. HDLC ratio

Fig.5.4: Alcohol Intake, Total Chol., Tot. Chol./ HDLC ratio
At the end of the simulation, his Total cholesterol to HDLC ratio decreases to 5.39 from 5.9, and total cholesterol increases by $6.5 \mathrm{mg} / \mathrm{dL}$ out of which $4.9 \mathrm{mg} / \mathrm{dL}$ is in HDLC i.e. total cholesterol is $215.10 \mathrm{mg} / \mathrm{dl}$, HDLC is 39.9 and LDLC is 131.28 .

\section{Association of smoking and alcohol drinking}

Alcohol drinking and cigarette smoking are social habits of lifestyle that are closely related to a variety of diseases [19]. Smoking affects the blood lipid profile: serum HDL and LDL cholesterol concentrations have been reported to be lower and higher, respectively, in smokers than in nonsmokers [20][21]. On the other hand, moderate alcohol consumption is known to lower the incidence of coronary heart disease [22]. In this simulation, the subject is observed for 21 days, taking $50 \mathrm{~g}$ of wine and smoking 15 cigarette sticks per day. The results follow (figure 5.5 and figure 5.6):
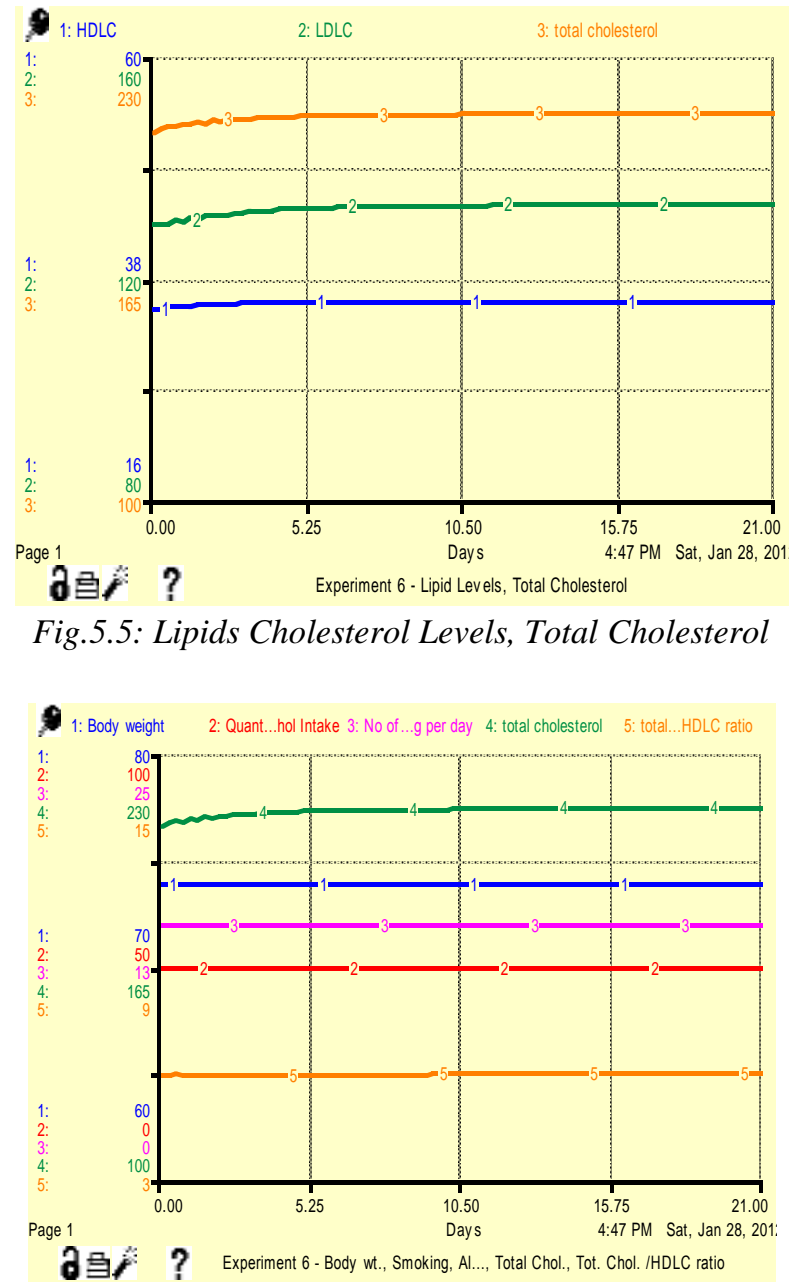

Fig.5.6: Alcohol Intake, Total Chol., Tot. Chol./ HDLC ratio

At the end of the simulation, his Total cholesterol to HDLC ratio decreases to 5.98 from 5.9 , total cholesterol is 213.98 $\mathrm{mg} / \mathrm{dl}, \mathrm{HDLC}$ is 35.77 and LDLC is 133.54 are very close

Page | 47 
the respective Base Level values. The purpose of this study was to investigate whether the effects of alcohol drinking on lipids cholesterol were influenced by cigarette smoking. A strong relationship between alcohol consumption and smoking has been verified as suggested in the paper [23].

\section{CONCLUSION}

The model is built using system dynamics methodology and it has a systemic view which takes two CVD risk factors Smoking and Alcohol Intake of the person into account. Our model successfully gave this result with its parameters. In the base equilibrium run, we considered borderline high blood cholesterol values of the subject. In subsequent simulations, it is confirmed that smoking increases LDLC and decreases HDLC levels, while moderate amount of alcohol intake increases HDLC level, however no significant changes has been found in LDLC level. The simulation results are in confirmation with other research paper in the field.

Medical decision support system for risk assessment of CVD due to Smoking and/or Alcohol Intake, using System Dynamics Simulation software (iThink V9.0.2 ), is designed, implemented and evaluated. The proposed framework would be particularly useful for researchers in the field but also for medical practitioners and developers of medical decision support systems.

\section{REFERENCES}

[1] Robert K. Murray, Daryl K. Granner and Victor W. Rodwell (eds), 2006, "Cholesterol Synthesis, Transport, \& Excretion", Harper's Illustrated Biochemistry, pp. 230-240, McGraw- Hill, New York.

[2] Rajagopal G. Cholesterol-an update. Biomed 2003; 23 : $1-8$.

[3] Haydar Al-Shalah, 2010, Effect of Light and Heavy Smoking on High Density Lipoprotein Cholesterol Level, Medical Journal of Babylon-Vol. 7- No. 4 -3.

[4] Whitehead T P, Robinson D, Allaway S L., 1996, the effects of cigarette smoking and alcohol consumption on blood lipids: Adose-related study on men. Annals of Clinical Biochemistry; 33: 99-106.

[5] Marda K, Noguchi Y, Fukui T., 2003, The effects from cessation from cigarette smoking on the lipid and lipoprotein profiles: a meta analysis. Preventive Med; 37: 283-90.

[6] Van der Gagg MS, Vantel A, Vermunt SH, Scheek LM, Schaafsma G, Hendricks HF., 2001, Alcohol consumption stimulates early steps in reverse transport of cholesterol. J Lipid Res; 42: 2077-83.

[7] Doll R., 1997, One for the heart. BMJ;315:1664-1668.
[8] Forrester JW., 1961, Industrial Dynamics. Cambridge, Mass: MIT Press.

[9] Forrester JW., 1969, Urban Dynamics. Cambridge, Mass: MIT Press.

[10] Forrester JW., 1971, Counterintuitive behavior of social systems. Technol Rev.;73:53-68.

[11] Forrester JW.,1980, Information sources for modeling the national economy. J Am Stat Assoc. 75:555-574.

[12]Forrester JW, Senge PM.,1980,Tests for building confidence in system dynamics models. In: System Dynamics, TIMS Studies in the Management Sciences. New York, NY: North-Holland; 209-228.

[13]Murray, K. R., 2006, "Cholesterol Synthesis, Transport, \& Excretion", in Robert K. Murray, Daryl K. Granner and Victor W. Rodwell (eds), Harper's Illustrated Biochemistry, pp. 230-240, McGraw- Hill, New York.

[14]Doll R, Peto R, Boreham J, Sutherland I., 2004, Mortality in relation to smoking: 50 years' observations on male British doctors.. BMJ; 328(7455) :1519-27.

[15] Ockene IS, Miller NH., 1997, Cigarette smoking, cardiovascular disease, and stroke: a statement for healthcare professionals from the American Heart Association. American Heart Association Task Force on Risk Reduction, Circulation. 96(9):3243-7.

[16] Grobbee, D. E., E. B. Rimm, U. Keil, and S. Renaud. 1999. Alcohol and cardiovascular system. In Health Issues Related to Alcohol Consumption. E. I. MacDonald, editor. ILSI Europe, Blackwell Science, Philadelphia, PA. 125-179.

[17] Yin et al., 2007, "Comparison of demography, diet, lifestyle, and serum lipid levels between the Guangxi Bai Yao and Han populations", Journal of Lipid Research, Volume 48,

[18] Hendriks, H. F. J., J. Veenstra, A. van Tol, J. E. M. Groener, and G. Schaafsma. 1998. Moderate doses of alcoholic beverages with dinner and postprandial high density lipoprotein composition. Alcohol Alcohol. 33: 403-410.

[19] I. Wakabayashi, 2008, "Associations of alcohol drinking and cigarette smoking with serum lipid levels in healthy middle-aged men", Alcohol \& Alcoholism Vol. 43, No. 3, pp. 274-280.

[20] Whitehead, T. P., Robinson, D. and Allaway, S. L., 1996, The effects of cigarette smoking and alcohol consumption on blood lipids: A dose-related study on men. Annals of Clinical Biochemistry 33, 99-106.

[21]Lee, K. S., Park, C. Y., Meng, K. H. et al. ,1998, The association of cigarette smoking and alcohol consumption with other cardiovascular risk factors in

Page | 48 
men from Seoul, Korea. Annals of Epidemiology 8, 31-38.

[22] Corrao, G., Rubbiati, L., Bagnardi, V., Zambon, A. and Poikolainen, K. ,2000, Alcohol and coronary heart disease: A meta-analysis. Addiction 95, 1505-1523.

[23] Veenstra, J., Schenkel, J. A. and van Erp-Baart, A. M. et al.,1993, Alcohol consumption in relation to food intake and smoking habits in the Dutch National Food Consumption Survey. European Journal of Clinical Nutrition 47, 482-489.

[24] Ma, Hongbao, 2006, "Cholesterol and Human Health", The Journal of American Science, Vol. 2, pp. 46-50.

[25] Bayer, S., Koberle-Gaiser, M. and Barlow, J., 2007, Planning for Adaptability in Healthcare Infrastructure. Proceedings of the 25th International System Dynamics, 29 Jul-2 Aug 07, Boston, USA. System Dynamics Society. ISBN 978-0-9745329-7-4.

[26] Cody, J., Cavana, R.Y. and Pearson, D. ,2007, Social Structure and health : Preliminary work on a System Dynamics Concept Model. Proceedings of the 25th International System Dynamics, 29 Jul-2 Aug 07, Boston, USA. System Dynamics Society. ISBN 978-09745329-7-4.

[27] Haslett, T., 2007, From Hospital Plans to Computer Simulation : A Case study of Alfred Centre. Proceedingsof the 25th International System Dynamics, 29 Jul-2 Aug 07, Boston, USA. System Dynamics Society. ISBN 978-0-9745329-7-4.

[28] Chu, S.C., Kin, C.K. and Lam, S.S. ,2003, Hospital lift system simulator: A performance evaluator-predictor. European Journal of Operational Research, 146: 1:156-180.

[29] Hethcote, H.W., Horby, P. and Mcintyre p., 2004, Using computer simulationsto compare pertussis vaccination strategies in Australia. Vaccine journal,22(17-18):2181-91. 